\title{
Bayesian competing risks analysis without data stratification
}

\author{
Atanu Bhattacharjee \\ Centre for Cancer Epidemiology, The Advanced Centre for Treatment, Research and Education in Cancer (ACTREC), Tata Memorial Centre, (An Autonomous Institution of \\ the Department of Atomic Energy(DAE), Government of India), Navi Mumbai, 410210, India
}

A R T I C L E I N F O

\section{Keywords:}

Bayesian

Cause-specific survival

Event-time

MCMC

\begin{abstract}
A B S T R A C T
Background: The Cox Proportional Hazard ( $\mathrm{CPH}$ ) model is a commonly used method to study death as the outcome of therapeutic effect. However, death may occur due to other causes not as the direct therapeutic effect. Death due to other causes is known as competing risks (CR). The conventional strategy is to handle the CR inflated death data with the stratified $\mathrm{CPH}$ model. However, it reduces the power of the data analysis. It is important to establish a model that can handle the CR without stratifying the data. This paper is devoted to developing the CR model.

Objective: Develop a statistical model that can handle death data analysis without stratifying in the presence of CR.

Methods: Statistical methodology is established to deal with CR. The Bayesian Inference is considered to overcome the computational difficulties. The Markov chain Monte Carlo (MCMC) was used to run the model. Model is illustrated for lung adenocarcinoma patient's data. The arm wise comparison on adenocarcinoma treated patients is performed. The computation of the competing risk model is performed in open source software OpenBugs.

Results: The proposed method helps to obtain the posterior estimates of different covariates with credible intervals. The posterior mean (SD) estimate helps to decide the best effective treatment through survival analysis in presences of CR.

Conclusions: This work is helped to work with CR data without stratifying it. It provides flexibility to analyze death data with CR by Bayesian.
\end{abstract}

\section{Introduction}

The primary focus of any oncology therapeutic effect study is too prolonged the duration of survival. The conventional survival analysis assumed that Competing Risk (CR) is not present in the dataset. This ignorance of CR may incorporate the misleading statistical inference about the therapeutic arm. ${ }^{1-4}$ It may happen that the CR is independent in nature as a cause of the therapeutic effect. The only available procedure is to discard the independent CR patient's data from survival analysis. It helps to avoid the biased inference in the clinical decision. ${ }^{5,6}$ However, discarding the patient's data can reduce the power of the study. In some studies, very minimal therapeutic effect benefit is expected. In this scenario, it becomes very crucial to explore the causes of death.

The deep exploration of the causes of death among treated patients gives scope for an in-depth comparison between treated arms. It is helpful to establish a decision among best effective therapy. The study can be assembled through consideration of all the patients into alive or dead. The causes of death could be disease progression or something else. Therefore the cause of death not related to therapeutic effect is called as competing risks (CR).

A comprehensive review of CR analysis can be found in Crowder. ${ }^{7,8}$ There is an application on statistical analysis with CR data for system failure information. ${ }^{9}$ Recently, there is also an attempt to develop the CR model through Bayesian inference with incomplete trial information. ${ }^{10}$ Literature also supports about how to monitor the CR. ${ }^{11}$ The choice of prior distribution for CR analysis is also documented. ${ }^{12}$ The Bayesian analysis of CR data is explored through Weibull with the same shape and different scale parameters. ${ }^{13}$ However, there is not any document about how to work with CR analysis for Phase III randomized controlled trial data. This work is dedicated towards exploring the application of CR analysis in the context of randomized controlled trial.

\section{Data methodology}

This study makes use of publicly available of a clinical trial data which is carried out to characterize the performance of different prognostic indices on gene expression for 442 lung adenocarcinomas.

E-mail address: atanustat@gmail.com. 
The data is available at NCBI Gene Expression Omnibus (GEO) data repository under accession number GSE68465 (http://www.ncbi.nlm. nih.gov/geo). The performance of prognostic models for 442 lung adenocarcinoma was tested through a large training-testing, multi-site, blinded validation study. ${ }^{14}$ The performance of a prognostic model is measured through gene expression data set in the combination of pathological and clinical outcomes. The relapse-free and overall survival duration for all patients is measured. As an intention to treat, a few patients have selected for adjuvant chemotherapy and some of them are not. This study provides the comparison of overall survival between adjuvant and non-adjuvant chemotherapy.

However, it failed to account the $\mathrm{CR}$ by conventional survival analysis. In such situations, it is required to compare the efficacy of adjuvant chemotherapy through deep consideration of occurrence of death and accommodating different competing risks, which requires the development of models that consider both endpoints simultaneously. All patients are coded as status $=1$ (If they died) or 0 (If they alive) at the end of the study in publicly available data sheet.

But in this work, patients died, followed by the relapse of the disease are defined as death due to therapeutic failure and coded as status $=1$. Patients followed as relapse-free but died are defined as CR and coded as status $=2$. Patients those are alive are defined as status $=0$. The competing event is separated in this manner. It is expected that this proposed model can support further survival analysis for eventual clinical utilization.

Further, we developed a novel Bayesian framework for the survival analysis of CR inflated data. The proposed method is developed without stratifying the patient's data. A Markov model is considered to support the computational complexity. The frequentist approach to make the inference is considered. This proposed method is developed to deliver the scientific community with a tool to investigate the CR data in a randomized clinical trial.

\section{A framework for survival analysis}

It is obvious for a scientist who is not aware of CR but whose research involves time-to-event or time-to-death data. The analysis of such data is called as survival analysis. The event needs not to be death always. It can be the distant relapse or loco-regional relapse etc. Survival analysis is well developed and can be easily implemented in all statistical software. However, there are some situations where conventional survival analysis is not appropriate enough to analysis the time-to-death data.

The objective in survival analysis is to measure the time between the dates of diagnosis or treatment initiation to the date of death. The basic assumption of conventional survival analysis through Kaplan-Meier is that the censored observations are 'independent'. ${ }^{2}$ It helps us to further assumed that the patients who are censored at a certain time point should be representative for those who are at risk at a specific time point. The "cumulative incidence function(CIF)" is defined as probability $F(t)$ of dying before time. ${ }^{15}$ If the time of death is measured for each individual then $F(t)$ can be estimated through the relative frequency of survival times less than $t$. The function $F(t)$ is estimated by Kaplan-Meier estimator as.

$1-\hat{S}(t)$ at time point $t$. The factor at failure time $S(t)$ is defined as $1-\frac{d_{i}}{n_{i}}$ where $d_{i}$ is death of the individual at time point $i$ and $n_{i}$ is the patients at risk at time point $i$.

The conventional survival analysis is not appropriate in presence of competing risks. It is required to adapt with caution. The Kaplan-Meier estimation is a method of estimating the survival probability at different time points. In presence of CR, Kaplan-Meier cannot be considered with probability. Their complement (1-KM) is defined as a probability of an event of interest in the real world where other types of events do not exist. ${ }^{16}$

$\mathrm{CR}$ concerns the situation where more than one cause of failure is possible. ${ }^{16-19}$ It is assumed that all the failure time distributions are continuous. In the right censored, individual $i$ is assumed to have an event time $T_{i}$ and a censoring time $C_{i}$, observation are $T_{i}=\min \left(T_{i}, C_{i}\right)$ and $\delta_{i}=I\left(T_{i} \leq C_{i}\right)$, if $\delta_{i}=1$, it shows that $t_{i}$ is observed otherwise not. Let the event time and censoring time denoted as $\left(T_{i}, C_{i}\right)$ and $T_{i} \sim S$ for $S(t)=\operatorname{prob}(T>t)$, and having censoring distribution $C_{i}$. At any point of time, the individual who are censored can be presented by those who remain under observation.

\section{A framework for Bayesian competing risks data analysis}

There are some dedicated packages in widely used statistical software. Preliminary analysis of our randomized controlled trial data was performed using the $\mathrm{R}$ (version 3.4.0), and the cmprsk (version 2.2-7) package. The cumulative incidence was plotted through cuminc function (Fig. 3).

Similarly, in SAS the \%CIF macro is helpful to estimate the cumulative incidence functions.

The conventional Cox PH model is unavoidable when lost to followup is nil in a long-term follow-up study. It can be accomplished with Fine-Gray model to handle competing risk events.

The nonparametric estimation through CIF is also helpful to deal with competing risks. The widely adopted approach to handle eventime data are Cox proportional hazard ( $\mathrm{CPH}$ ) modeled. However, it is not suitable tool to handle the event-time data merged with competing risks of the event. It considers the either the competing risk as censored observation or death due to the treatment effect. Therefore, the choice to work with $\mathrm{CPH}$ for competing risk data is the application of the cause specific sub-hazard function.

However, it is not suitable enough in terms of direct interpretation of survival probability. The other limitation of $\mathrm{CPH}$ is that the data should follow the proportionality assumption. If it failed to follow the proportionality assumption, then stratified $\mathrm{CPH}$ is suitable to work with non-proportional hazard. However, it reduces the power of study due stratification of the entire sample size. It is better to consider dedicated approach to analysis event-time data having competing risks of event of interest.

The CR is defined as the situation in which an individual can experience more than one type of event. ${ }^{20}$ It is also defined as the failure to achieve independence between the time of an event and the censoring mechanism. ${ }^{21}$ The situation where one type of event either preclude the occurrence of another event under investigation or fundamentally alters the probability of occurrence of this other event. ${ }^{22}$ Let $\mathrm{C}$ is censoring variable. The duration of observation is specified by $\mathrm{T}$. The survival data can be represented as a bivariate random variable (C,T). If $\mathrm{C}=1$, then $\mathrm{T}$ is the time at which the event occurred. If $\mathrm{C}=0$, then $\mathrm{T}$ is time at which the observation was censored. Further, $\mathrm{C}$ can be extended into $0, i(i=1, p)$. If $C=0$ then the observation is censored, and $C=i$ then observation is not censored. The term $i$ is considered to define death due to different reasons. If death is due to treatment failure then it can be considered as $i=1$. If death is due to other causes then it is considered as $\mathrm{i}=2$. In this analysis, we will restrict with the maximum value for $i$ as 2 .

$\lambda_{p}\left(t_{i}\right)=\lim _{\delta t \rightarrow 0} \frac{\operatorname{Pr}\left(t_{i}<T \leq t_{i}, C_{i}=p \mid T>t_{i}\right)}{\delta t}$

The cumulative cause-specific hazard is defined as

$\Delta_{p}(t)=\int_{0}^{t} \lambda_{p}(s) d s$

The cause specific survival function is defined as

$S_{p}(t)=\exp \left(-\left(\Delta_{p}(t)\right)\right.$

The overall survival function is defined as 
$S(t)=\exp \left(\sum_{p=1}^{P}-\Delta_{p}(t)\right)$

The cause-specific density or sub-density function

$f_{p}\left(t_{i}\right)=\lim _{\delta t \rightarrow 0} \frac{\operatorname{Pr}\left(t_{i}<T<t_{i}+\delta t, C_{i}=p\right)}{\delta t}$

Let the censoring indicator for the ith individual's is defined as $\delta_{i p}=1$ if $C_{i}=p \varepsilon(1,2, \ldots P)$ and $\delta_{i p}=0$ otherwise.

The covariates are defined as $X_{i p}$ with the proportional hazard assumption as

$\lambda_{p}\left(t_{p} \mid X_{i p}, \beta_{p}\right)=h_{0 p}\left(t_{i}\right) \exp \left(\beta_{p} X_{i p}\right)$

and the likelihood function is defined as

$L_{i}=f_{p}\left(t_{i} \mid X_{i p}, \beta_{p}\right) \prod_{k \neq p} S\left(t_{i} \mid X_{i k}, \beta_{k}\right)$

Further, the hazard function is extended as

$h_{i}=\left(t_{i} \mid b_{i p}, X_{i p}, \beta\right) h_{0 p}\left(t_{i}\right) \exp \left(\beta_{p} X_{i p}+b_{i p}\right)$

$b_{i p} \sim N(0, \Sigma)$

to accommodate the cause specific frailty effects.

This analysis is performed through open source software OpenBugs (www.openbugs.net). OpenBugs is free available software. The OpenBugs code is included as Annexure at the end of this paper.

\section{Analysis and results}

Table 1 gives the descriptive statistics for patients in the study sample. Baseline characters observed as continuing variable are summarized through the median, while binary variables with frequencies and percentages. Fig. 1 show that the Kaplan-Meier estimates of overall survival in patients treated with Adjuvant and Non-Adjuvant arms. The median overall survival of the patients treated Adjuvant and Non-Adjuvant arms were 47 and 70.5 months respectively. The Log-rank test value on overall survival comparison is obtained with p-value 0.0015 . Initially, the cumulative incidence rates of death among patients are plotted. Further, the Kaplan-Meier curves without and with considering

Table 1

Baseline characteristics of patients in the Adjuvant vs Non-adjuvant chemotherapy $(\mathrm{N}=443)$.

\begin{tabular}{|c|c|c|c|}
\hline Parameter & Adjuvant(N = 89) & Non-Adjuvant( $\mathrm{N}=354)$ & All Patients \\
\hline \multicolumn{4}{|l|}{ Age } \\
\hline Mean & 61.61 & 65.12 & 64.41 \\
\hline Range & $36-82$ & $33-87$ & $33-87$ \\
\hline \multicolumn{4}{|l|}{ Gender } \\
\hline Male & $40(44.94 \%)$ & $183(51.69 \%)$ & $223(50.34 \%)$ \\
\hline Female & $49(55.06 \%)$ & $171(48.31 \%)$ & $220(49.66 \%)$ \\
\hline \multicolumn{4}{|c|}{ Tobacco Consumption } \\
\hline Smoker & $73(82.08 \%)$ & $227(64.12 \%)$ & $300(67.72 \%)$ \\
\hline Non-Smoker & $16(17.8 \%)$ & $127(35.88 \%)$ & $143(32.28 \%)$ \\
\hline \multicolumn{4}{|l|}{ Disease Stage } \\
\hline N0T1 & $5(5.62 \%)$ & $109(30.79 \%)$ & $114(25.73 \%)$ \\
\hline NOT2 & $34(38.2 \%)$ & $128(36.16 \%)$ & $162(36.57 \%)$ \\
\hline N0T3 & $2(2.5 \%)$ & $14(3.95 \%)$ & $16(3.61 \%)$ \\
\hline N0T4 & $0(0 \%)$ & $7(1.98 \%)$ & $7(1.58 \%)$ \\
\hline N1T1 & $4(4.29 \%)$ & $20(5.65 \%)$ & $24(5.42 \%)$ \\
\hline N1T2 & $21(26.6 \%)$ & $34(9.60 \%)$ & $55(12.42 \%)$ \\
\hline N1T3 & $1(1.12 \%)$ & $6(1.69 \%)$ & $7(1.58 \%)$ \\
\hline N1T4 & $0(0 \%)$ & $2(0.56 \%)$ & $2(0.45 \%)$ \\
\hline N2T1 & $4(4.49 \%)$ & $7(1.98 \%)$ & $11(2.48 \%)$ \\
\hline N2T2 & $14(15.73 \%)$ & $20(5.65 \%)$ & $34(7.67 \%)$ \\
\hline N2T3 & $1(1.12 \%)$ & $4(1.13 \%)$ & $5(1.13 \%)$ \\
\hline N2T4 & $2(2.25 \%)$ & $1(0.28 \%)$ & $3(0.68 \%)$ \\
\hline NXT4 & $0(0 \%)$ & $1(0.28 \%)$ & $1(0.23 \%)$ \\
\hline Unknown & $1(1.12 \%)$ & $1(0.28 \%)$ & $2(0.45 \%)$ \\
\hline
\end{tabular}

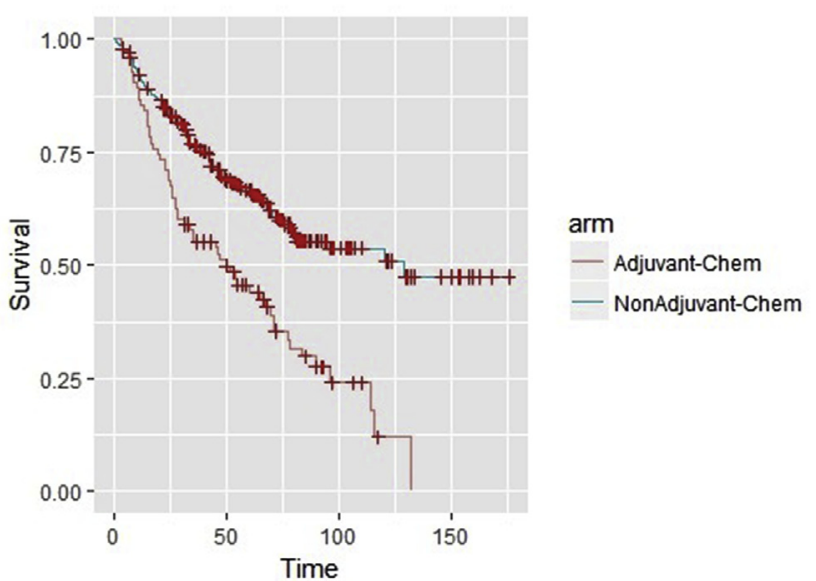

Fig. 1. Kaplan-Meier Curve: Without considering competing risk.

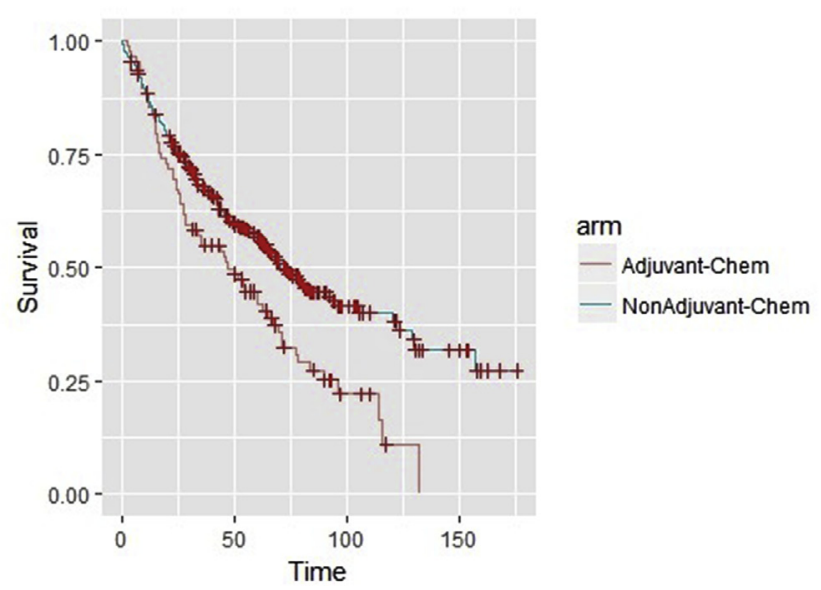

Fig. 2. Kaplan-Meier Curve: by considering competing risk as event.

the CR are plotted in Figs. 1 and 2 respectively. The duration of followup for both the curves is considered on overall survival. A total of 20,000 iterations with inference from the last 1 s5000 iterations are considered. The posterior mean(SD) and 95\% credible intervals of regression parameters are given in Table 2 . It shows that Age is a positive risk factor for both the causes (i.e. death and CR).However, the impact of age for cause of CR is comparatively less(0.151) in comparison to cause as death (0.355). The arms are defined as (arm $=1$ for Adjuvant and arm $=2$ for Non-Adjuvant).

Table 2

Posterior estimates of the parameters observed through competing risk analysis.

\begin{tabular}{lll}
\hline Parameter & Posterior Mean(SD) & $(2.5 \%, 97.5 \%)$ \\
\hline Intercept & & \\
$\left(\beta_{01}\right)$ & $6.034(2.623)$ & $(2.429,12.18)$ \\
$\left(\beta_{02}\right)$ & $-8.802(3.483)$ & $(-15.96,-2.22)$ \\
Disease Stage & & \\
$\left(\beta_{11}\right)$ & $-0.113(0.361)$ & $(-0.878,0.545)$ \\
$\left(\beta_{12}\right)$ & $0.606(1.168)$ & $(-1.546,3.049)$ \\
Age & & \\
$\left(\beta_{21}\right)$ & $0.355(0.484)$ & $(-0.536,1.368)$ \\
$\left(\beta_{22}\right)$ & $0.151(0.658)$ & $(-1.118,1.479)$ \\
Arm & & \\
$\left(\beta_{31}\right)$ & $-0.054(0.050)$ & $(-0.190,-0.002)$ \\
$\left(\beta_{32}\right)$ & $0.054(0.044)$ & $(-0.027,0.137)$ \\
Smoking & & \\
$\left(\beta_{41}\right)$ & $-0.045(0.019)$ & $(-0.086,-0.007)$ \\
$\left(\beta_{42}\right)$ & $0.027(0.056)$ & $(-0.057,0.159)$ \\
\hline
\end{tabular}




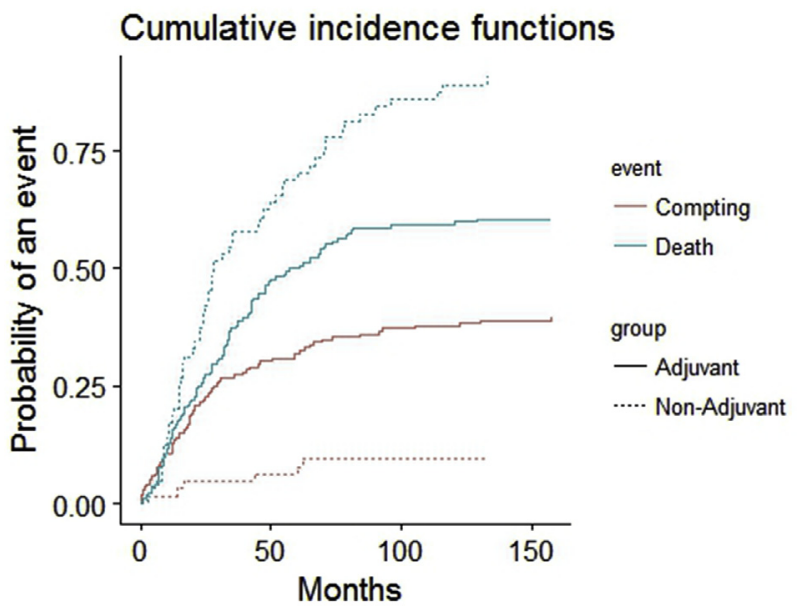

Fig. 3. Cumulative incidence function.

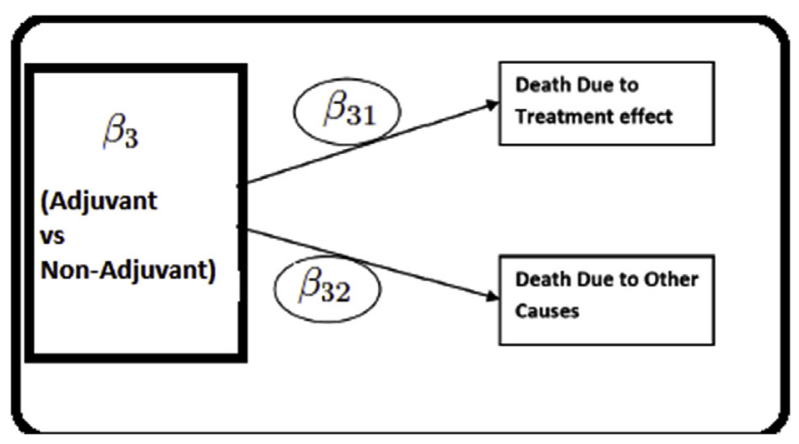

Fig. 4. Competing risk frame.

The MCMC output provides the posterior mean estimate $\beta_{31}$ for Non-Adjuvant arm in comparison to Adjuvant arm. The $\beta_{31}$ is -0.054 with standard deviation as 0.050 . The $95 \%$ corresponding credible interval is observed with $(-0.190,-0.002)$. This $\beta_{31}$ gives the inference for cohort of individuals those are shifted from censoring to death status during the study. The negative estimates of $\beta_{31}$ represents that the NonAdjuvant arm significantly prolongs the duration of survival in comparison to Adjuvant arm. However, $\beta_{32}$ provides the not significant estimates about CR. The weekly arm was found better in comparison to Non-Adjuvant arm for occurrence of CR. The CR framework is presented in Fig. 4. Similar trend is also observed for the smoking status also. The regression coefficients interval for smoking status was significant for causes of death but not significant for causes with CR.

\section{Discussion}

The CR precludes the occurrence of the primary event of interest. The application of CIF provides better estimates in comparison to Kaplan-Meier survival estimates in this context. Fitting regression model in the presence of CR can be handled by (I) Modeling the effect of covariates on the cause-specific hazard of the outcome or (II) Modeling the effect of covariates with CIF. However, the cause-specific hazard considers the subsets of those individuals are event free. But CIF provides the best estimate of the effect of covariates on the absolute risk of the outcome over time. During CR analysis the cause of failures could be independent or dependent in nature. The presence of covariates is used to provide information about the type's dependence between causes of death. There is an attempt to explore the distribution assumption of causes of death 7 and model the CR through the latent failure time assumption 13. Under parametric setup with Weibull distribution, the CR modeling is explored. ${ }^{23}$ Till now the majority of methods is developed through the frequentist approach, mainly by a non-parametric procedure. But there is limited literature available on Bayesian CR modeling.The copula is found suitable to deal with CR problem. ${ }^{24}$ However, it failed to consider the covariates. There is an attempt on Bayesian by the multistate model as well as as. ${ }^{25-29}$ The Bayesian inference is found satisfactory for semi-parametric risk data analysis in cross-over trial. ${ }^{30}$ It is attempted for lifetime risk modeling on the non-terminal event.

\section{Conclusion}

However, there is a very rare occurrence of a non-terminal event in any trial. We have illustrated the application of the $\mathrm{CR}$ model into the randomized controlled trial to serve the basic objective. The basic objective is to find the best effective therapy, i.e. Adjuvant or NonAdjuvant. It is also possible to perform regression with cause-specific models by R. But thus reduce the power of the study. There are some dedicated packages like "timereg", "cmprsk", "msate" to deal with CR in open source software R. These are built to carry with cumulative incidence function. But there is limited code available to deal with CR with Bayesian inference. The Bayesian can be considered as an alternative to computing the CR model. The result was further compared with the frequentist approach. It was also found similar trends in results. However, Bayesian provides a more robust and flexible interpretation. It is also possible to perform regression with cause-specific models by R. But thus reduce the power of the study as well. It is requested to write to the corresponding author about running this code with other suitable data.

This work is an extension of the Bayesian counterpart in the CR model. The tool like CPH and Kaplan-Meier estimates are well developed. However, it is not advised to adopt these tools due to their application limitations. It is required to explore the reasons for the deaths of cancer treated patients. It is incorrect to consider death due to other causes as censored. Similarly, it cannot be considered as death due to treatment failure as well. However, both the consideration makes a positive or negative impact on inference. The existing methods (like CIF or others) only dealt with different causes of death through subsetting the survival dataset. Therefore, the inference drawn from subset data is not acceptable, due to the inadequate power of the study. It becomes challenging to deal with different causes of death without subsetting the data. This work is an extension of Bayesian Inference with CR data without stratifying. We attempted the CR modeling without subsetting the dataset. It is useful for CR inflated survival data analysis. OpenBugs software is used to perform the analysis. The estimation is carried out by Markov chain Monte Carlo (MCMC) method.

\section{Declarations of interest}

The authors declare no conflict of interest. 


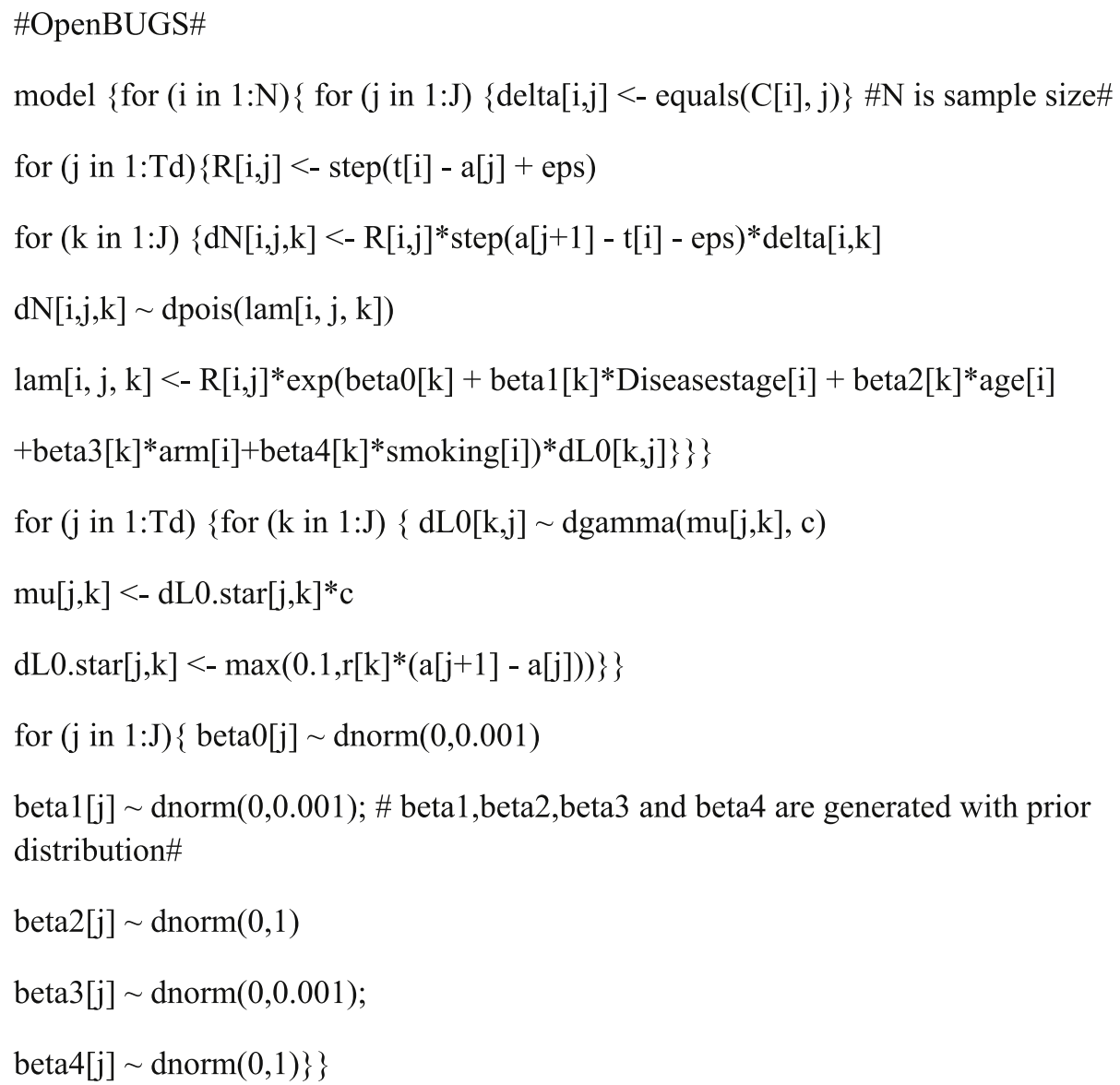

\section{Appendix A. Supplementary data}

Supplementary data to this article can be found online at https://doi.org/10.1016/j.cegh.2019.08.010.

\section{References}

1. Putter H, Fiocco M, Geskus RB. Tutorial in biostatistics: competing risks and multistate models. Stat Med. 2007;26:2389-2430.

2. Lau B, Cole SR, Gange SJ. Competing risk regression models for epidemiologic data. Am J Epidemiol. 2009;170:244-256.

3. Satagopan JM, Ben-Porat L, Berwick M, Robson M, Kutler D, Auerbach AD. A note on competing risks in survival data analysis. Br J Canc. 2004;91:1229-1235.

4. Berry SD, Ngo L, Samelson EJ, Kiel DP. Competing risk of death: an important consideration in studies of older adults. J Am Geriatr Soc. 2010;58:783-787.

5. Tsiatis AA. An example of nonldeutifiability in competing risks. Scand Actuar J. 1978:235-239 1978.

6. Pepe MS, Mori M. Kaplan-meier, marginal or conditional probability curves in summarizing competing risks failure time data? Stat Med. 1993;12:737-751.

7. Crowder MJ. Classical Competing Risks. Chapman and Hall/CRC; 2001.

8. Conklin J. Classical competing risks. Technometrics. 2002;44 298-298.

9. Sen A, Basu S, Banerjee M. Ch. 19. Analysis of masked failure data under competing risks. Handbook of Statistics. Elsevier; 2001:523-540.

10. Bhattacharjee A. Bayesian competing risks model: an application to breast cancer clinical trial with incomplete observations. J Stat Manag Syst. 2015;18:381-404.

11. Steiner SH, MacKay RJ. Monitoring processes with data censored owing to competing risks by using exponentially weighted moving average control charts. J R Stat Soc Ser C Appl Stat. 2001;50:293-302.

12. Lin DKJ, Usher JS, Guess FM. Bayes estimation of component-reliability from masked system-life data. IEEE Trans Reliab. 1996;45:233-237.

13. Kundu D, Pradhan B. Bayesian analysis of progressively censored competing risks data. Sankhya B. 2011;73:276-296.

14. Director's Challenge Consortium for the Molecular Classification of Lung Adenocarcinoma. Shedden K, Taylor JMG, Enkemann SA, Tsao M-S, Yeatman TJ, et al. Gene expression-based survival prediction in lung adenocarcinoma: a multi- site, blinded validation study. Nat Med. 2008;14:822-827.

15. Olsen J, Christensen K, Murray J, Ekbom A. An Introduction to Epidemiology for Health Professionals. New York, NY: Springer; 2010.

16. Jenkins M, Flynn A, Smart T, et al. A statistician's perspective on biomarkers in drug development. Pharm Stat. 2011;10:494-507.

17. Bhattacharyya $\mathrm{T}$, Bhattacharjee $\mathrm{A}$. Competing risk: an illustration with aspiration pneumonia in head and neck cancer patients undergoing radical radiotherapy: a biostatistician's perspective. Indian J Cancer. 2014;51:406-409.

18. Klein JP. Modelling competing risks in cancer studies. Stat Med. 2006;25:1015-1034.

19. Holt JD. Competing risk analyses with special reference to matched pair experiments. Biometrika. 1978;65:159-165.

20. Kalbfleisch JD, Prentice RL. Competing Risks and Multistate Models. The Statistical Analysis of Failure Time Data. John Wiley \& Sons, Inc.; 2002:247-277.

21. Arriagada R, Rutqvist LE, Kramar A, Johansson H. Competing risks determining event-free survival in early breast cancer. Br J Canc. 1992;66:951-957.

22. Gooley TA, Leisenring W, Crowley J, Storer BE. Estimation of failure probabilities in the presence of competing risks: new representations of old estimators. Stat Med. 1999;18:695-706

23. Rao BR, Talwalker S, Kundu D. Confidence intervals for the relative risk ratio parameter from survival data under a random censorship model in biomedical and epidemiologic studies (by simulation). Biom J. 1991;33:959-984.

24. Fu H, Wang Y, Liu J, Kulkarni PM, Melemed AS. Joint modeling of progression-free survival and overall survival by a Bayesian normal induced copula estimation model. Stat Med. 2013;32:240-254.

25. Sharples LD. Use of the Gibbs sampler to estimate transition rates between grades of coronary disease following cardiac transplantation. Stat Med. 1993;12:1155-1169.

26. Bayesian Semiparametric Multi-State Models. Techn. Univ., Sonderforschungsbereich Statistische Analyse Diskreter Strukturen. 2006; 2006.

27. van den Hout A, Fox J-P, Klein Entink RH. Bayesian inference for an illness-death model for stroke with cognition as a latent time-dependent risk factor. Stat Methods Med Res. 2015;24:769-787. 
28. van den Hout A, Matthews FE. Estimating dementia-free life expectancy for Parkinson's patients using Bayesian inference and microsimulation. Biostatistics. 2009;10:729-743.

29. Pan S-L, Wu H-M, Yen AM-F, Chen TH-H. A Markov regression random-effects model for remission of functional disability in patients following a first stroke: a Bayesian approach. Stat Med. 2007;26:5335-5353

30. Zhang $\mathrm{Y}$, Chen M-H, Ibrahim JG, et al. Bayesian gamma frailty models for survival data with semi-competing risks and treatment switching. Lifetime Data Anal. 2014;20:76-105. 